\title{
Non-alcoholic fatty liver disease in the first trimester and subsequent development of gestational diabetes mellitus
}

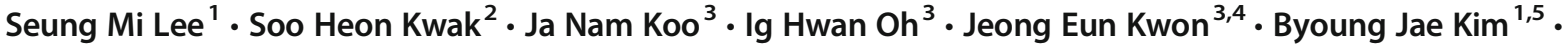 \\ Sun Min Kim ${ }^{1,5}$. Sang Youn Kim ${ }^{6}$. Gyoung Min Kim ${ }^{7}$. Sae Kyung Joo ${ }^{8,9}$. Bo Kyung Koo ${ }^{8,9}$. Sue Shin ${ }^{10,11}$. \\ Chanthalakeo Vixay ${ }^{12}$. Errol R. Norwitz ${ }^{13}$. Chan-Wook Park ${ }^{1}$. Jong Kwan Jun ${ }^{1}$ - Won Kim ${ }^{8,9}$. Joong Shin Park ${ }^{1}$
}

Received: 10 July 2018 / Accepted: 10 October 2018 / Published online: 23 November 2018

(C) Springer-Verlag GmbH Germany, part of Springer Nature 2018

\begin{abstract}
Aims/hypothesis Although there is substantial evidence that non-alcoholic fatty liver disease (NAFLD) is associated with impaired glucose homeostasis, the clinical significance of NAFLD in pregnant women has not been well determined. This study investigates the relationship between NAFLD in the first trimester and the subsequent development of gestational diabetes mellitus (GDM).

Methods A multicentre, prospective cohort study was conducted in which singleton pregnant Korean women were assessed for NAFLD at 10-14 weeks using liver ultrasound, fatty liver index (FLI) and hepatic steatosis index (HSI). Maternal plasma adiponectin and selenoprotein P concentrations were measured. Participants were screened for GDM using the two-step approach at 24-28 weeks.

Results Six hundred and eight women were included in the final analysis. The prevalence of NAFLD was 18.4\% (112/608) and 5.9\% (36/608) developed GDM. Participants who developed GDM had a higher prevalence of radiological steatosis $(55.6 \%$ vs $16.1 \% ; p<0.001)$ and higher FLI ( 40.0 vs $10.7 ; p<0.001)$ and HSI ( 35.5 vs $29.0 ; p<0.001)$. The risk of developing GDM was significantly increased in participants with NAFLD and was positively correlated with the severity of steatosis. This relationship
\end{abstract}

Won Kim and Joong Shin Park contributed equally to this work.

Electronic supplementary material The online version of this article (https://doi.org/10.1007/s00125-018-4779-8) contains peer-reviewed but unedited supplementary material, which is available to authorised users.

Joong Shin Park

jsparkmd@snu.ac.kr

Won Kim

drwon1@snu.ac.kr

1 Department of Obstetrics and Gynecology, Seoul National University College of Medicine, 101 Daehak-ro,

Seoul, Jongno-gu 03080, South Korea

2 Department of Internal Medicine, Seoul National University College of Medicine, Seoul, South Korea

3 Seoul Women's Hospital, Incheon, South Korea

4 Department of Obstetrics and Gynecology, Hallym University Sacred Heart Hospital, Gyeonggi-do, South Korea

5 Department of Obstetrics and Gynecology, Seoul Metropolitan Government Seoul National University Boramae Medical Center, Seoul, South Korea

6 Department of Radiology, Seoul National University College of Medicine, Seoul, South Korea
7 Department of Radiology, Yonsei University College of Medicine, Seoul, South Korea

8 Department of Internal Medicine, Seoul National University College of Medicine, Seoul, South Korea

9 Division of Gastroenterology and Hepatology, Department of Internal Medicine, Seoul Metropolitan Government Seoul National University Boramae Medical Center, 20, Boramae-ro 5-gil, Seoul, Dongjak-gu 07061, South Korea

10 Department of Laboratory Medicine, Seoul National University College of Medicine, Seoul, South Korea

11 Department of Laboratory Medicine, Seoul Metropolitan Government Seoul National University Boramae Medical Center, Seoul, South Korea

12 Department of Obstetrics and Gynecology, University of Health Sciences, Vientiane, Lao PDR

13 Department of Obstetrics and Gynecology, Tufts University School of Medicine, Boston, MA, USA 


\section{Research in context}

\section{What is already known about this subject?}

- There is substantial evidence that non-alcoholic fatty liver disease (NAFLD) is associated with impaired glucose homeostasis

- The clinical significance of NAFLD in pregnant women has not been fully established

What is the key question?

- Is NAFLD in pregnant women associated with gestational diabetes mellitus (GDM)?

What are the new findings?

- The risk of developing GDM was significantly increased in women with NAFLD

- Maternal plasma adiponectin and selenoprotein P levels were correlated with both NAFLD severity and the risk of developing GDM

How might this impact on clinical practice in the foreseeable future?

- $\quad$ NAFLD in early pregnancy can be considered to be an independent risk factor for GDM

between NAFLD and GDM remained significant after adjustment for metabolic risk factors, including measures of insulin resistance. Maternal plasma adiponectin and selenoprotein P levels were also correlated with both NAFLD severity and the risk of developing GDM.

Conclusions/interpretation NAFLD in early pregnancy is an independent risk factor for GDM. Adiponectin may be a useful biomarker for predicting GDM in pregnant women.

Keywords Gestational diabetes mellitus · Insulin resistance $\cdot$ Non-alcoholic fatty liver disease $\cdot$ Prediction

$\begin{array}{ll}\text { Abbreviations } \\ \text { ALT } & \text { Alanine aminotransferase } \\ \text { AST } & \text { Aspartate aminotransferase } \\ \text { FLI } & \text { Fatty liver index } \\ \text { GCT } & \text { Glucose challenge screening test } \\ \text { GDM } & \text { Gestational diabetes mellitus } \\ \text { GGT } & \gamma \text {-Glutamyl transferase } \\ \text { HSI } & \text { Hepatic steatosis index } \\ \text { ICD } & \text { International Classification of Diseases } \\ \text { NAFLD } & \text { Non-alcoholic fatty liver disease }\end{array}$

\section{Introduction}

Non-alcoholic fatty liver disease (NAFLD) is characterised histologically by hepatic fat accumulation in individuals without excessive alcohol consumption. It is one of the most common chronic liver diseases in both eastern and western countries, and its prevalence is reported to be $10-40 \%$ in adult populations, depending on age, lifestyle and race/ethnicity [1-4]. There is substantial evidence that NAFLD is associated with impaired glucose tolerance, type 2 diabetes and the metabolic syndrome [5-7], although the molecular mechanisms underlying these associations are not clear. Interestingly, the relationship between NAFLD, obesity and insulin resistance appears to vary depending on an individual's race/ethnicity [4]. Asian individuals with NAFLD have a lower BMI than those in western countries, and a significant proportion have low insulin resistance [8-10]. Taken together, these data suggest that Asian populations may have a different genetic or environmental susceptibility to NAFLD [4, 8, 11].

We hypothesise that NAFLD may be a risk factor for the development of gestational diabetes mellitus (GDM). Several reports have shown an association between a history of GDM and NAFLD in non-pregnant women [12-15]. Although recent studies have suggested a possible relationship between NAFLD and GDM [16], this has not been systematically examined and there is a paucity of information on this relationship in Asian populations.

In the current study, we examined the association between radiological and/or biochemical evidence of NAFLD in early pregnancy and the subsequent risk of developing GDM in pregnant Korean women. Moreover, since it is already known that adiponectin and selenoprotein $\mathrm{P}$ are likely to be responsible for linking hepatic steatosis to impaired glucose homeostasis $[17,18]$, we investigated whether circulating levels of 
these cytokines are associated with NAFLD severity in pregnant women and can be used to predict the subsequent risk of developing GDM.

\section{Methods}

Study design We prospectively enrolled singleton pregnant women presenting for prenatal care before 14 weeks of gestation at Incheon Seoul Women Hospital and Seoul Metropolitan Government Seoul National University Boramae Medical Center in Seoul, Korea, from the ongoing 'Fatty Liver in Pregnancy' registry (ClinicalTrials.gov registration no. NCT 02276144). All participants gave written informed consent before enrolment. General clinical and demographic information was collected, including maternal age, parity, pre-gestational diabetes, a prior history of GDM, a familial history of diabetes among first-degree relatives, pre-gestational height, weight, waist circumference, alcohol consumption during pregnancy using the validated cut-annoyed-guilty-eye questionnaire [19], and a history of chronic liver disease such as hepatitis B or hepatitis C, autoimmune hepatitis, primary biliary cholangitis, primary sclerosing cholangitis, haemochromatosis and Wilson's disease. Women with pre-existing chronic liver disease or pregestational diabetes were excluded from the analysis.

At 10-14 weeks of gestation, the presence or absence of NAFLD was evaluated by means of liver ultrasound, the fatty liver index (FLI) and the hepatic steatosis index (HSI) (see below). All participants were screened for the presence of GDM using the two-step approach at 24-28 weeks. The study was approved by the Institutional Review Board of Seoul Metropolitan Government Seoul National University Boramae Medical Center and the Public Institutional Review Board designated by Ministry of Health and Welfare.

Definition and severity of NAFLD based on liver ultrasonography The presence of NAFLD was defined as the detection of bright echogenic patterns within the liver detected by ultrasonography. A semiquantitative grading system (grade 0-3) was adopted to assess the degree of hepatic steatosis, as described elsewhere [20, 21]. Liver ultrasonography was performed by experienced sonographers at the time of routine fetal ultrasonography. B-mode imaging of the liver was stored for subsequent review using a standardised protocol, which included a single image capture of the liver and adjacent right kidney. Hard copies of liver ultrasound images were reviewed by two experienced radiologists (S. Y. Kim and G. M. Kim) who were blinded to clinical and biochemical data of the study participants at the time of examination. If there was a discrepancy in the interpretation of the liver ultrasounds, a final diagnosis was made by consensus after a detailed discussion.
Risk stratification of NAFLD based on non-invasive steatosis indices Participants were asked to fast for at least $8 \mathrm{~h}$ before the examination and venous blood was taken at the time of liver ultrasonography. The FLI was calculated according to the equation listed below, which incorporated BMI, waist circumference and circulating levels of triacylglycerols and $\gamma$ glutamyl transferase (GGT) [22].

$\mathrm{FLI}=\left(\exp \left[\operatorname{Model}_{\mathrm{FLI}}\right]\right) /\left(1+\exp \left[\operatorname{Model}_{\mathrm{FLI}}\right]\right) \times 100$

where $\operatorname{Model}_{\mathrm{FLI}}=\left(0.953 \times \log _{e}\right.$ [triacylglycerol $(\mathrm{mmol} / \mathrm{l}) \times$ $88.5])+\left(0.139 \times\right.$ BMI $\left.\left[\mathrm{kg} / \mathrm{m}^{2}\right]\right)+\left(0.718 \times \log _{e}\right.$ GGT [IU/ 1]) $+(0.053 \times$ waist circumference $[\mathrm{cm}])-15.745$

Study participants were classified into three groups according to the FLI $[23,24]$ : low-risk group (FLI $\leq 20$ ); intermediaterisk group $(20<\mathrm{FLI}<60)$ and high-risk group (FLI $\geq 60)$. The HSI was calculated according to the following equation [25]:

$$
\begin{aligned}
\mathrm{HSI} & =8 \times \mathrm{ALT}(\mathrm{IU} / \mathrm{l}) / \mathrm{AST}(\mathrm{IU} / \mathrm{l})+\mathrm{BMI}\left(\mathrm{kg} / \mathrm{m}^{2}\right) \\
& +2(\text { if } \mathrm{T} 2 \mathrm{D})+2(\text { if } \mathrm{F})
\end{aligned}
$$

where ALT is alananine aminotransferase, AST is aspartate aminotransferase, T2D is type 2 diabetes and F is female sex.

The participants were classified into three groups according to the HSI: low risk of steatosis (HSI <30); intermediate risk $(30 \leq \mathrm{HSI} \leq 36)$ and high risk (HSI $>36)$.

\section{Determination of insulin resistance and measurement of cir-} culating adiponectin and selenoprotein $\mathbf{P}$ concentrations Venous blood samples were collected when participants were in the fasting state and at the time of liver ultrasonography and serum glucose levels were measured. The venous blood samples were then centrifuged at $2000 \mathrm{~g}$ for $10 \mathrm{~min}$ and aliquoted for storage at $-70^{\circ} \mathrm{C}$ until assay. The stored serum was assayed in batches for insulin levels. Fasting serum glucose levels were measured using an enzymatic method (Glucose HK; Roche diagnostics, Indianapolis, IN, USA) using a Roche/Hitachi 911 (Roche diagnostics) chemistry analyser. Serum insulin concentrations were determined with an immunometric assay (IRMA) in duplicate using the same batch of a kit (INS-Irma; DIAsource ImmunoAssays, Louvain-la-Neuve, Belgium). Systemic insulin resistance was evaluated using the HOMAIR, as previously described [26]. Stored plasma samples were also assayed for adiponectin $(\mu \mathrm{g} / \mathrm{ml})$ and selenoprotein $\mathrm{P}$ $(\mu \mathrm{g} / \mathrm{ml})$ using commercially available enzyme-linked immunosorbent assay kits (from R\&D Systems, Minneapolis, MN, USA and Cloud-Clone, Houston, TX, USA, respectively).

Screening and diagnosis of GDM All participants were screened for the presence of GDM using the two-step approach at 24-28 weeks according to the recommendations of the American College of Obstetricians and Gynecologists 
[27]. For the $50 \mathrm{~g}$ oral glucose challenge screening test (GCT), serum glucose levels were measured $1 \mathrm{~h}$ after a $50 \mathrm{~g}$ oral glucose load in the non-fasting state. A serum glucose level of $\geq 7.8 \mathrm{mmol} / \mathrm{l}$ was regarded as a positive GCT. For women with a positive screening GCT, a follow-up $100 \mathrm{~g}$ oral glucose tolerance test was performed. A diagnosis of GDM required two or more elevated glucose levels (i.e. $\geq 5.3 \mathrm{mmol} / 1$ for fasting glucose, $\geq 10 \mathrm{mmol} / 1$ for $1 \mathrm{~h}$ glucose, $\geq 8.6 \mathrm{mmol} / 1$ for $2 \mathrm{~h}$ glucose and $\geq 7.8 \mathrm{mmol} / \mathrm{l}$ for $3 \mathrm{~h}$ glucose) [28].

Statistical analysis Proportions were compared using the $\chi^{2}$ test or Fisher's exact test, where appropriate. Comparison of continuous variables between groups was performed using the independent $t$ test, the Mann-Whitney $U$ test, ANOVA or the Kruskal-Wallis test. Spearman's correlation analysis was used to assess the relationship between the FLI and circulating adiponectin and selenoprotein P levels. To determine which factors are independently associated with GDM, a binary logistic regression model was generated. After adjustment for clinical and biological confounding factors, the association between NAFLD and GDM was assessed by multiple logistic regression with Firth's penalised likelihood to avoid bias in variable estimates due to a small sample size [29].

A prior sample size calculation was performed to determine how many pregnant women would be needed to detect an increased risk for GDM from 5\% to $15 \%$ in women without and with NAFLD, respectively. We estimated the frequency of NAFLD would be 10-20\% in women of reproductive age according to previous reports $[30,31]$. With $80 \%$ power and a type 1 error of $5 \%$, we determined that we would require 443 women with a frequency of $20 \%$ of NAFLD and would require 744 women with a frequency of $10 \%$ of NAFLD.

A $p$ value of $<0.05$ was considered statistically significant. SPSS version 21.0 software (IBM, Armonk, NY, USA) was used for the analysis.

\section{Results}

Study population A total of 678 pregnant women were recruited and underwent liver ultrasonography at 10-14 weeks of gestation between November 2014 and September 2016. Among them, 34 women (four with viral hepatitis, nine with pregestational diabetes and 21 who did not have a fasting blood sampled collected at the time of liver ultrasonography) were excluded from the final analysis. Moreover, results of GDM screening tests were not available in 36 women. Of these, two participants underwent termination of pregnancy before GDM testing, 11 had incomplete glucose test results, 19 were lost to follow-up and four gave informed consent at enrolment but withdrew consent during the follow-up period. The remaining 608 participants were included in the final analysis.
Clinical characteristics according to GDM status Among the study population, 5.9\% (36/608) developed GDM. Table 1 presents the baseline clinical, anthropometric, biochemical and metabolic characteristics and obstetric outcomes of the study population by GDM status. Study participants who developed GDM had a higher rate of a prior history of GDM and higher BMI, waist circumference and systolic and diastolic blood pressure (BP) at 10-14 weeks than normoglycaemic pregnant women, indicating an unfavourable metabolic status that pre-dated the development of GDM. In addition, participants who developed GDM had higher levels of AST and ALT, triacylglycerol, GGT, fasting glucose, insulin and HOMA-IR already at 10-14 weeks of pregnancy compared with those who did not develop GDM. In this study population, the OR $(95 \% \mathrm{CI})$ of fasting insulin and HOMA-IR was $1.165(1.113,1.220)$ and $1.936(1.588,2.631)$, respectively. In terms of delivery outcomes, as expected, participants with GDM had a higher rate of large-for-gestational-age (LGA) neonates than those with normal glucose tolerance.

Radiological features and non-invasive steatosis indices according to GDM status At 10-14 weeks of gestation, the prevalence of NAFLD was $18.4 \%$ (112/608) based on liver ultrasonography (14.1\% with grade 1 steatosis; $4.3 \%$ with grade 2 or 3 steatosis) and $28.5 \%(173 / 608)$ based on the FLI (23.1\% with intermediate-risk FLI; 5.3\% with high-risk FLI). Table 2 compares the results from liver ultrasonography and non-invasive steatosis indices (FLI and HSI) at 10-14 weeks of gestation between participants who did and did not develop GDM. Women who developed GDM had a higher rate of fatty liver based on liver ultrasonography at 10-14 weeks of gestation and also had higher FLI and HSI values than those who did not develop GDM. Indeed, women with fatty liver at 10-14 weeks were more likely to go on to develop GDM, and the risk of developing GDM was increased according to the radiological severity of NAFLD: the incidence of GDM was $3.2 \%$ in women with grade 0 steatosis, $10.5 \%$ in those with grade 1 steatosis and $42.3 \%$ in those with grade 2 or 3 steatosis $\left(p<0.001, \chi^{2}\right.$ for trend; Fig. 1a). The risk of GDM was also increased in women with higher FLI and HSI $\left(p<0.001\right.$ for both, $\chi^{2}$ for trend; Fig. $1 \mathrm{~b}, \mathrm{c})$. Interestingly, the risk of GDM was also increased according to the FLI and HSI, even after confining the analysis to women without radiological evidence of steatosis: $1.8 \%$ in women with FLI $\leq 20,6.8 \%$ in women with $20<$ FLI $<60$, $28.6 \%$ in women with FLI $\geq 60\left(p<0.001, \chi^{2}\right.$ for trend $)$; $1.9 \%$ in women with HSI $<30,4.8 \%$ in women with $30 \leq$ HSI $\leq 36,8.1 \%$ in women with HSI $>36,\left(p<0.05, \chi^{2}\right.$ for trend $)$.

The relationship between radiological evidence of NAFLD and GDM As shown in Table 3, in the age-adjusted analysis (model 1), radiological evidence of NAFLD as assessed by liver ultrasonography was significantly associated with the development of GDM (OR 6.66 [95\% CI 3.36, 13.41]; $p<0.001$ ). 
Table 1 Baseline clinical, anthropometric, biochemical and metabolic features and pregnancy outcomes of the study population

\begin{tabular}{|c|c|c|c|}
\hline Characteristic & $\begin{array}{l}\text { No GDM } \\
(n=572)\end{array}$ & $\begin{array}{l}\text { GDM } \\
(n=36)\end{array}$ & $p$ value \\
\hline \multicolumn{4}{|l|}{ Baseline characteristics before pregnancy } \\
\hline Nulliparity & $301(52.6)$ & $20(55.6)$ & 0.864 \\
\hline Family history of diabetes & $123(21.5)$ & $11(30.6)$ & 0.215 \\
\hline History of prior GDM & $13(2.3)$ & $4(11.1)$ & 0.015 \\
\hline Hypertension & $2(0.4)$ & $1(2.8)$ & 0.168 \\
\hline BMI, $\mathrm{kg} / \mathrm{m}^{2}$ & $21.2(19.4-23.4)$ & $25.3(21.8-29.0)$ & $<0.001$ \\
\hline Waist circumference, $\mathrm{cm}$ & $71(68-74)$ & $76(71-81)$ & $<0.001$ \\
\hline \multicolumn{4}{|c|}{ Baseline characteristics at the time of liver ultrasonography ${ }^{a}$} \\
\hline $\begin{array}{l}\text { Maternal age at the time of liver } \\
\text { ultrasonography, years }\end{array}$ & $32(30-34)$ & $33(31-34)$ & 0.479 \\
\hline Gestational age, weeks & $12.4(12.1-12.9)$ & $12.4(12.1-12.7)$ & 0.664 \\
\hline BMI, $\mathrm{kg} / \mathrm{m}^{2}$ & $21.7(19.8-23.8)$ & $26.4(23.0-29.0)$ & $<0.001$ \\
\hline Waist circumference, $\mathrm{cm}$ & $82.0(76.8-88.0)$ & $93.8(85.0-101.8)$ & $<0.001$ \\
\hline Systolic BP, mmHg & $111(104-119)$ & $118(110-129)$ & 0.004 \\
\hline Diastolic BP, mmHg & $67(61-72)$ & $69(65-80)$ & 0.006 \\
\hline \multicolumn{4}{|c|}{ Biochemical and metabolic features at the time of liver ultrasonography ${ }^{\mathrm{a}}$} \\
\hline AST, U/1 & $16(14-19)$ & $18(15-22)$ & 0.047 \\
\hline ALT, U/1 & $11(8-14)$ & $16(10-27)$ & 0.001 \\
\hline Cholesterol, mmol/1 & $4.4(3.96-4.92)$ & $4.58(4.04-5.18)$ & 0.220 \\
\hline HDL-cholesterol, mmol/1 & $1.68(1.44-1.92)$ & $1.57(1.20-1.84)$ & 0.046 \\
\hline LDL cholesterol, mmol/1 & $2.15(1.80-2.53)$ & $2.14(1.68-2.56)$ & 0.825 \\
\hline Triacylglycerol, mmol/1 & $1.22(0.96-1.54)$ & $1.74(1.45-2.19)$ & $<0.001$ \\
\hline GGT, U/1 & $12(10-15)$ & $17(11-24)$ & 0.001 \\
\hline Fasting glucose, mmol/l & $4.27(3.89-4.61)$ & $4.55(4.05-5.05)$ & 0.001 \\
\hline Insulin, pmol/1 & $56.3(36.8-77.8)$ & $106.3(58.3-154.9)$ & $<0.001$ \\
\hline HOMA-IR & $1.5(1.0-2.2)$ & $2.9(1.7-5.1)$ & $<0.001$ \\
\hline \multicolumn{4}{|l|}{ Pregnancy outcome ${ }^{\mathrm{b}}$} \\
\hline Gestational age at delivery, weeks & $39.3(38.4-40.0)$ & $38.6(38.0-39.7)$ & 0.071 \\
\hline Birthweight at delivery, $\mathrm{kg}$ & $3.22(2.99-3.48)$ & $3.20(2.92-3.62)$ & 0.788 \\
\hline Large-for-gestational-age neonates & $55(9.9)$ & $8(22.9)$ & 0.024 \\
\hline
\end{tabular}

Data are presented as $n(\%)$ or median (interquartile range)

${ }^{a}$ Liver ultrasonography was performed at 10-14 weeks of gestation

${ }^{\mathrm{b}}$ For pregnancy outcome, $n=555$ (no GDM) and 35 (GDM)
This association persisted after additional adjustment for metabolic risk factors (prior history of GDM, greater waist circumference and high BP at 10-14 weeks [defined as high systolic or diastolic BP]; model 2), although it was attenuated (OR 3.63 [95\% CI 1.65, 7.96]; $p<0.005$ ). As insulin resistance plays an important role in the progression of NAFLD, an additional adjustment for HOMA-IR was performed (model $3)$. This did not significantly reduce the OR for this association (OR 3.28 [95\% CI 1.49, 7.22]; $p<0.005$ ). Because adiponectin and selenoprotein $\mathrm{P}$ levels are known to link hepatic steatosis to impaired glucose tolerance, multivariate analysis was performed adjusting for adiponectin and selenoprotein P levels (model 4). This association persisted in this model but was slightly attenuated (OR 2.50 [95\% CI 1.07, 5.77]; $p<0.05)$.
We adjusted for BMI instead of waist circumference in the multivariate analysis. NAFLD was a significant risk factor for GDM even after adjustment for the following variables: (1) $\mathrm{BMI}$ at the time of liver ultrasonography; (2) age, prior history of GDM, BMI at the time of liver ultrasonography, high BP at the time of liver ultrasonography and (3) age, prior history of GDM, BMI at the time of liver ultrasonography, high BP at the time of liver ultrasonography and HOMA-IR.

The relationship between steatosis severity (as assessed by the FLI or HSI) and the development of GDM Multivariate logistic regression analysis was conducted to determine the effect of steatosis severity at 10-14 weeks of gestation on the likelihood of developing GDM (Table 3). In the ageadjusted analysis (model 1), steatosis severity as assessed by 
Table 2 Radiological findings and non-invasive steatosis indices at 10-14 weeks of gestation

\begin{tabular}{|c|c|c|c|}
\hline Characteristic & $\begin{array}{l}\text { No GDM } \\
(n=572)\end{array}$ & $\begin{array}{l}\text { GDM } \\
(n=36)\end{array}$ & $p$ value \\
\hline \multicolumn{3}{|l|}{ Steatosis determined by liver ultrasonography } & \multirow[t]{3}{*}{$<0.001$} \\
\hline Grade 0 steatosis & $480(83.9)$ & $16(44.4)$ & \\
\hline Grade 1 steatosis & $77(13.5)$ & $9(25.0)$ & \\
\hline Grade 2 or 3 steatosis & $15(2.6)$ & $11(30.6)$ & $<0.001$ \\
\hline $\begin{array}{l}\text { Presence of fatty liver determined by liver } \\
\text { ultrasonography }\end{array}$ & $92(16.1)$ & $20(55.6)$ & $<0.001$ \\
\hline \multirow{2}{*}{\multicolumn{3}{|c|}{$\begin{array}{l}\text { FLI at the time of liver ultrasonography }{ }^{a} \\
\text { Risk subgroups based on the FLI }\end{array}$}} & $<0.001$ \\
\hline & & & \multirow[t]{4}{*}{$<0.001$} \\
\hline Low risk of steatosis, FLI $\leq 20$ & $425(75.0)$ & $7(19.4)$ & \\
\hline Intermediate risk of steatosis, $20<\mathrm{FLI}<60$ & $123(21.7)$ & $16(44.4)$ & \\
\hline High risk of steatosis, FLI $\geq 60$ & $19(3.4)$ & $13(36.1)$ & \\
\hline HSI at the time of liver ultrasonography ${ }^{\mathrm{b}}$ & $29.0(26.8-32.5)$ & $35.5(32.0-39.5)$ & $<0.001$ \\
\hline \multicolumn{3}{|l|}{ Risk subgroups based on the HSI } & \multirow[t]{4}{*}{$<0.001$} \\
\hline Low risk of steatosis, HSI $<30$ & $336(59.2)$ & $6(16.7)$ & \\
\hline Intermediate risk of steatosis, $30 \leq \mathrm{HSI} \leq 36$ & $175(30.8)$ & $15(41.7)$ & \\
\hline High risk of steatosis, HSI $>36$ & $57(10.0)$ & $15(41.7)$ & \\
\hline \multicolumn{4}{|l|}{$\begin{array}{l}\text { Circulating biomarkers at the time of liver } \\
\text { ultrasonography }{ }^{c}\end{array}$} \\
\hline Adiponectin, $\mu \mathrm{g} / \mathrm{ml}$ & $5.34(3.13-8.28)$ & $1.95(1.32-2.93)$ & $<0.001$ \\
\hline Selenoprotein $\mathrm{P}, \mu \mathrm{g} / \mathrm{ml}$ & $5.83(2.90-14.51)$ & $10.01(6.72-34.86)$ & $<0.005$ \\
\hline
\end{tabular}

Data are presented as $n(\%)$ or median (IQR)

${ }^{\text {a }}$ For FLI at the time of liver ultrasonography, $n=567$ (no GDM) and 36 (GDM)

${ }^{\mathrm{b}}$ For HSI at the time of liver ultrasonography, $n=568$ (no GDM) and 36 (GDM)

${ }^{\mathrm{c}}$ For circulating biomarkers at the time of liver ultrasonography, $n=571$ (no GDM) and 36 (GDM) the FLI was significantly associated with GDM (OR 1.05 [95\% CI] 1.04, 1.07; $p<0.001)$. This association persisted after additional adjustment for metabolic risk factors (a prior history of GDM and waist circumference and high BP; model 2) (OR $1.07[95 \% \mathrm{CI}] 1.04,1.10 ; p<0.001)$. Moreover, the association persisted even after adjustment for HOMA-IR and circulating adiponectin and selenoprotein P levels (models 3 and 4). Similarly, steatosis severity as assessed by the HSI at 10-14 weeks of gestation was also significantly associated with subsequent GDM after multiple adjustments for metabolic risk factors, HOMA-IR and maternal plasma adiponectin and selenoprotein P levels (models 1-4; Table 3).
The relationship between maternal plasma adiponectin and selenoprotein P levels, NAFLD and GDM Pregnant women with NAFLD had significantly lower plasma concentrations of adiponectin and higher plasma concentrations of selenoprotein $\mathrm{P}$ than those without NAFLD and there appeared to be a stepwise relationship with more disparate levels in women with grade 2 or 3 steatosis (Fig. 2a, b). Maternal plasma adiponectin levels were inversely correlated with the FLI (Spearman's $\rho=-0.345, p<0.001$; Fig. 3a) and selenoprotein $\mathrm{P}$ concentrations were positively correlated with the FLI (Spearman's $\rho=0.258, p<0.001$; Fig. 3b). In particular, women who subsequently developed GDM had a

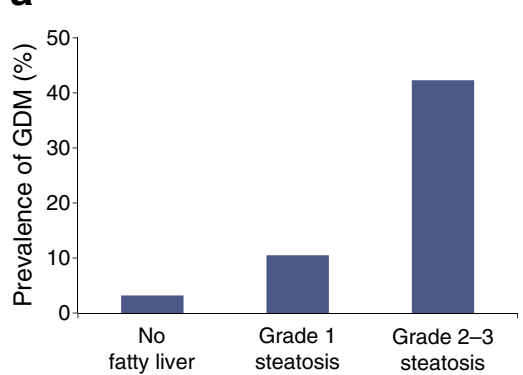

b

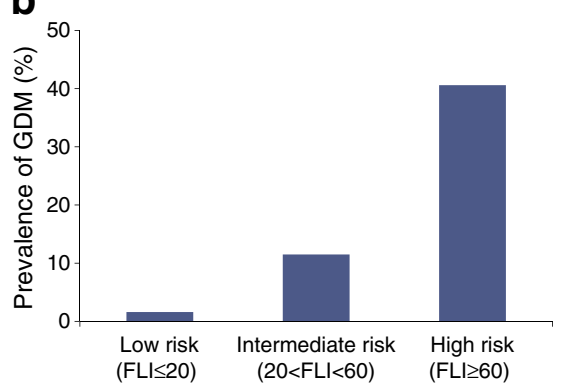

C

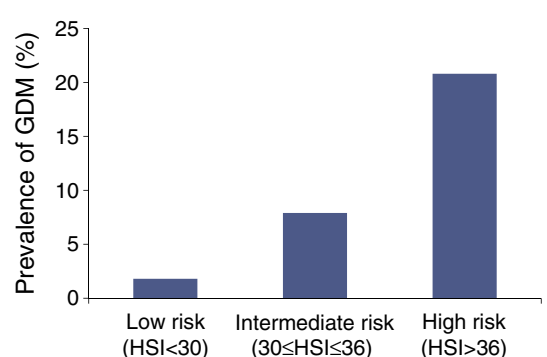

Fig. 1 The prevalence of GDM according to (a) the radiological severity of NAFLD $\left(p<0.001, \chi^{2}\right.$ for trend), (b) the NAFLD risk subgroup based on the FLI $\left(p<0.001, \chi^{2}\right.$ for trend) and (c) the NAFLD risk subgroup based on the HSI $\left(p<0.001, \chi^{2}\right.$ for trend) 
Table 3 Univariate and multivariate analyses producing ORs of risk factors for GDM

\begin{tabular}{|c|c|c|}
\hline Variable & OR $(95 \% \mathrm{CI})$ & $p$ value \\
\hline \multicolumn{3}{|l|}{ Fatty liver } \\
\hline Unadjusted & $6.45(3.26,12.97)$ & $<0.001$ \\
\hline Model 1 & $6.66(3.36,13.41)$ & $<0.001$ \\
\hline Model 2 & $3.63(1.65,7.96)$ & $<0.005$ \\
\hline Model 3 & $3.28(1.49,7.22)$ & $<0.005$ \\
\hline Model 4 & $2.50(1.07,5.77)$ & $<0.05$ \\
\hline \multicolumn{3}{|l|}{ FLI } \\
\hline Unadjusted & $1.05(1.04,1.07)$ & $<0.001$ \\
\hline Model 1 & $1.05(1.04,1.07)$ & $<0.001$ \\
\hline Model 2 & $1.07(1.04,1.10)$ & $<0.001$ \\
\hline Model 3 & $1.06(1.04,1.10)$ & $<0.001$ \\
\hline Model 4 & $1.05(1.02,1.08)$ & $<0.005$ \\
\hline \multicolumn{3}{|l|}{ HSI } \\
\hline Unadjusted & $1.23(1.16,1.31)$ & $<0.001$ \\
\hline Model 1 & $1.23(1.16,1.31)$ & $<0.001$ \\
\hline Model 2 & $1.17(1.08,1.28)$ & $<0.001$ \\
\hline Model 3 & $1.16(1.06,1.26)$ & $<0.005$ \\
\hline Model 4 & $1.12(1.03,1.23)$ & $<0.05$ \\
\hline \multicolumn{3}{|c|}{ Selenoprotein $\mathrm{P}>90$ th percentile } \\
\hline Unadjusted & $4.09(1.82,8.64)$ & $<0.005$ \\
\hline Model 1 & $4.02(1.79,8.49)$ & $<0.001$ \\
\hline Model 2 & $3.18(1.29,7.35)$ & $<0.05$ \\
\hline Model 3 & $3.97(1.57,9.50)$ & $<0.005$ \\
\hline \multicolumn{3}{|c|}{ Adiponectin $<10$ th percentile } \\
\hline Unadjusted & $8.34(4.01,17.09)$ & $<0.001$ \\
\hline Model 1 & $8.36(4.02,17.14)$ & $<0.001$ \\
\hline Model 2 & $5.97(2.67,13.16)$ & $<0.001$ \\
\hline Model 3 & $5.09(2.25,11.36)$ & $<0.001$ \\
\hline
\end{tabular}

Model 1, adjusted for age; model 2, adjusted for a prior history of GDM, waist circumference at the time of liver ultrasonography, high systolic or diastolic BP at the time of liver ultrasonography in addition to model 1; model 3, adjusted for the HOMA-IR in addition to model 2; model 4, adjusted for adiponectin and selenoprotein $\mathrm{P}$ levels in addition to model 3 using cut-off for adiponectin $<10$ th percentile $(<1.72 \mu \mathrm{g} / \mathrm{ml})$ and for selenoprotein $\mathrm{P}>90$ th percentile $(>34.01 \mu \mathrm{g} / \mathrm{ml})$

significantly lower adiponectin and higher selenoprotein $\mathrm{P}$ concentrations at $10-14$ weeks than those who did not subsequently develop GDM (Fig. 2c, d). As the risk of GDM was associated with lower adiponectin and higher selenoprotein $\mathrm{P}$ plasma concentrations, women with lower adiponectin $(<10$ th percentile $[<1.72 \mu \mathrm{g} / \mathrm{ml}]$ ) or with higher selenoprotein $\mathrm{P}$ ( $>90$ th percentile $[>34.01 \mu \mathrm{g} / \mathrm{ml}]$ ) levels were designated as being at high risk of GDM. This relationship between lower adiponectin $(<10$ th percentile) and higher selenoprotein $\mathrm{P}$ ( $>90$ th percentile) levels and subsequent GDM remained significant even after adjustment for age, metabolic risk factors (prior history of GDM, higher waist circumference and high BP) and HOMA-IR (models 1-3; Table 3).
Next, we investigated whether adiponectin and selenoprotein P levels were associated with the development of GDM independently of NAFLD. Stratified analyses using medians of adiponectin and selenoprotein $\mathrm{P}$ levels provided additional discrimination for the risk of developing GDM (Fig. 4). Specifically, low adiponectin and high selenoprotein $P$ levels were independent predictors of GDM regardless of NAFLD status. Notably, a high prevalence of developing GDM was noted in women without NAFLD if their adiponectin levels were $<50$ th percentile; after adjusting for age, these women had a 5.4-fold higher risk of developing GDM than women who had no NAFLD but who had higher adiponectin levels (OR 5.44 [95\% CI 1.53, 19.36]; $p<0.01$ ) (see electronic supplementary material [ESM] Table 1). Similarly, a high prevalence of GDM was noted even in women without NAFLD if their selenoprotein P levels were $>50$ th percentile; after adjusting for age, these women had a 3.4-fold higher risk of developing GDM than women who had no NAFLD but who had lower selenoprotein P levels (OR 3.40 [95\% CI 1.08, 10.69]; $p<0.05$ ) (see ESM Table 2).

\section{Discussion}

In the current study, we demonstrate that the presence of NAFLD at 10-14 weeks of gestation - whether defined by ultrasound imaging or non-invasive steatosis indices such as the FLI and HSI - is associated with the subsequent development of GDM in a Korean population. This association persisted even after adjustment for metabolic risk factors, insulin resistance and circulating adipokine and hepatokine levels. Moreover, we observed a stepwise association between maternal plasma adiponectin and selenoprotein P levels and both the radiological and biochemical severity of NAFLD. The risk of subsequent GDM was also independently associated with adiponectin and selenoprotein $\mathrm{P}$ levels even after adjusting for metabolic variables and insulin resistance.

As the relationship between NAFLD, obesity and insulin resistance appears to vary according to race/ethnicity [8-10], it would be important to demonstrate the reproducibility of our results in non-Asian populations. Previous studies have suggested that women with a prior history of GDM are at increased risk of NAFLD [12-15]. To date, however, the clinical significance of NAFLD in pregnancy has not been fully established. A few recent studies investigated the risk of GDM in western women with NAFLD. The study by De Souza and colleagues from Canada suggested that the presence of NAFLD as assessed by liver ultrasound at 1114 weeks of gestation was significantly associated with a composite outcome defined as impaired fasting glucose, gestational impaired glucose tolerance or GDM at 24-28 weeks of gestation [32]. In a Swedish population-based study, 110 occurrences of pre-existing NAFLD before pregnancy were 

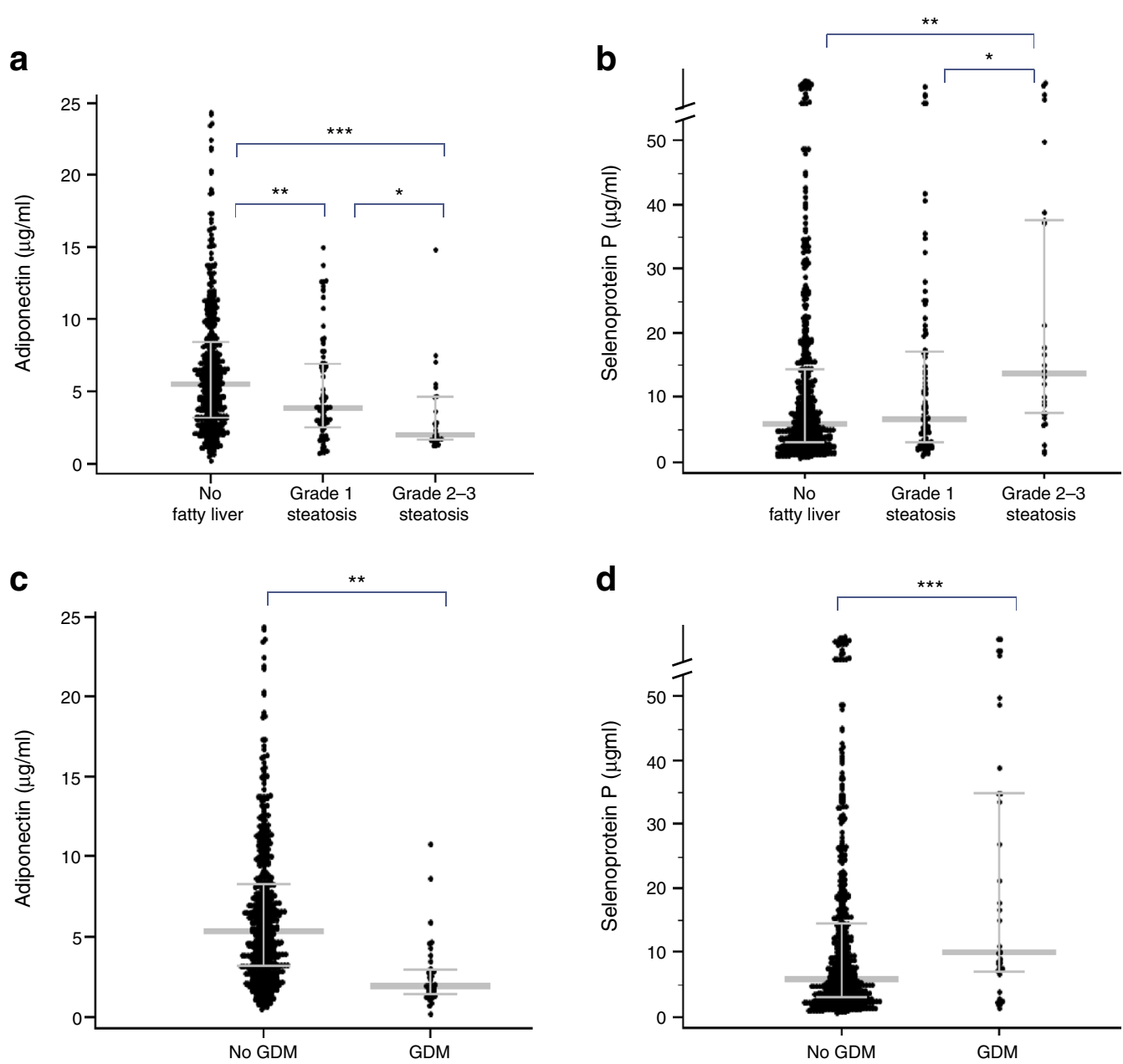

Fig. 2 Maternal plasma concentrations of adiponectin and selenoprotein $\mathrm{P}$ according to steatosis severity (a, b) and GDM status (c, d). $* p<0.05$; $* * p<0.005$ and ${ }^{* *} p<0.001$, Kruskal-Wallis test $(\mathbf{a}, \mathbf{b})$ or Mann-Whitney $U$ test $(\mathbf{c}, \mathbf{d})$. Horizontal grey lines indicate median and interquartile range

identified out of $1,960,416$ pregnant women with singleton pregnancy using International Classification of Diseases (ICD) codes [33]. Women with pre-existing NAFLD had an increased risk of developing GDM. However, in this report, the ICD-10 code for NAFLD seemed to capture a small subset of NAFLD, because only 110 women $(0.006 \%)$ were diagnosed with NAFLD among the 1,960,416 pregnant women. In an Egyptian study that included 200 pregnant women with NAFLD and 200 without, the risk of GDM was increased in women with vs without NAFLD [34]. However, to our knowledge, the current study is the first one to clearly demonstrate a significant association between fatty liver, as assessed by both radiological imaging and non-invasive steatosis indices, and the risk of GDM, although not a composite outcome.

In the current study, consistent with NAFLD diagnosed by ultrasound, women with abnormal FLI or HSI were also at significantly increased risk of developing GDM. The FLI is a multivariate model used to estimate fat accumulation in the liver and has been validated in multiple model systems [22, 24]. Given that sonographical estimation of fatty liver is largely subjective and examiner-dependent, the observation that the
FLI is also associated with the development of GDM reinforces our understanding of the clinical significance of NAFLD in pregnancy. Moreover, since ultrasound is not able to detect the accumulation of a small amount of fat in the liver and only yields a positive result when the liver fat content exceeds 30\% [35], non-invasive steatosis indices may be more useful as a predictive index in women with mild or moderate NAFLD. Indeed, in the current study, even when confining the statistical analysis to those women with a normal liver ultrasound, an abnormal FLI was still positively correlated with the risk of developing GDM. The risk of developing GDM varied across the FLI in a stepwise fashion, even in women who did not have NAFLD.

The mechanism by which NAFLD causes impaired glucose tolerance and increases the risk of developing GDM is not known. It is likely that the mechanism represents two different metabolic diseases that share a common metabolic dysfunction such as insulin resistance [32, 33]. However, in the current study, women with NAFLD were at increased risk of GDM even after adjustment for abdominal obesity and insulin resistance, although the statistical significance was 



Fig. 3 Correlation between FLI and (a) maternal plasma adiponectin levels (Spearman's $\rho=-0.345, p<0.001$ ) and (b) selenoprotein P levels (Spearman's $\rho=0.258, p<0.001$ ). Adiponectin, selenoprotein P and FLI were plotted as $\log _{e}$-transformed values

greatly attenuated after adjustment for HOMA-IR. These results suggest that mechanisms other than insulin resistance and obesity are likely to be involved. For example, proinflammatory cytokines originating from other intra-abdominal visceral tissues may be transferred to the liver, leading to fat accumulation and NAFLD [36, 37]. Thus, such release of adipokines and lipolytic activity make visceral adipose tissues more metabolically adverse, resulting in NAFLD and GDM $[38,39]$. Several adipokines and hepatokines have been implicated [40]. We previously reported that hepatic overexpression of activating transcription factor 3 (ATF3) plays an important role in oxidative stress-mediated hepatic steatosis and the development of type 2 diabetes in animal models of diabetes and in non-pregnant humans with NAFLD [41]. In the current study, we measured circulating levels of adiponectin and selenoprotein $\mathrm{P}$ at 10-14 weeks of gestation and showed that abnormal levels (low adiponectin or high selenoprotein $\mathrm{P}$ ) correlated with both NAFLD severity during early pregancy and the risk of GDM in the mid-gestation, suggesting that adiponectin may be a useful biomarker for predicting the development of GDM. Selenoprotein P seemed to be correlated with NAFLD and GDM but the levels of selenoprotein $P$ were higher only in women with grade 2 or 3 steatosis and the correlation coefficient was lower between selenoprotein P and FLI than between adiponectin and FLI. The correlation between adiponectin and GDM (and possibly the correlation between selenoprotein P and GDM) is consistent with the findings of a previous report showing an inverse correlation between adiponectin and selenoprotein $\mathrm{P}$ in individuals with type 2 diabetes [42]. Further mechanistic studies are needed to identify the mechanisms by which NAFLD leads to the development of GDM. In addition, the evaluation of liver ultrasound during the second or third trimester would enhance our understanding of the link between NAFLD and GDM, as steatosis is a dynamic process easily affected by weight gain and pregnancy is a state of altered metabolism.

From a clinical utility perspective, the risk of developing GDM is increased in pregnant women with NAFLD diagnosed at 10-14 weeks of gestation and this risk appears to increase in a stepwise fashion with the severity of NAFLD.

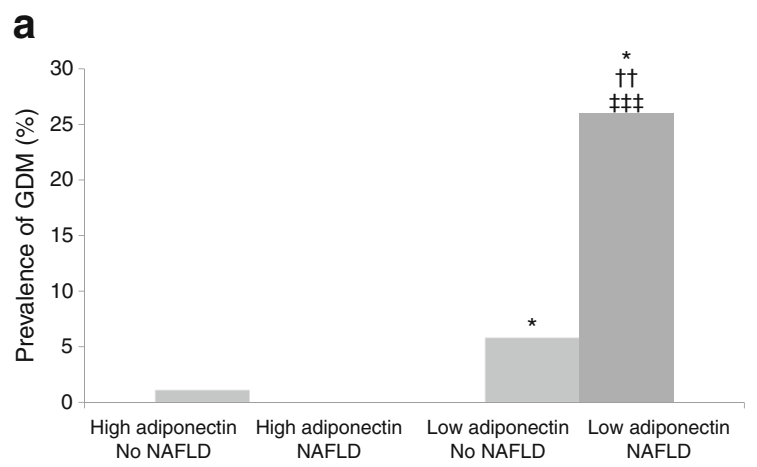

Fig. 4 The prevalence of GDM according to circulating adiponectin or selenoprotein P levels and NAFLD status. (a) The prevalence of GDM according to maternal plasma adiponectin and NAFLD status. High adiponectin is defined as $>50$ th percentile $(>5.02 \mu \mathrm{g} / \mathrm{ml})$ and low adiponectin is defined as $\leq 50$ th percentile. $* p<0.05 \mathrm{vs}$ high adiponectin without NAFLD; ${ }^{\dagger} p<0.005$ vs high adiponectin with NAFLD; $\$$ 林 $p<0.001$ vs low adiponectin without NAFLD; all Mann-Whitney $U$

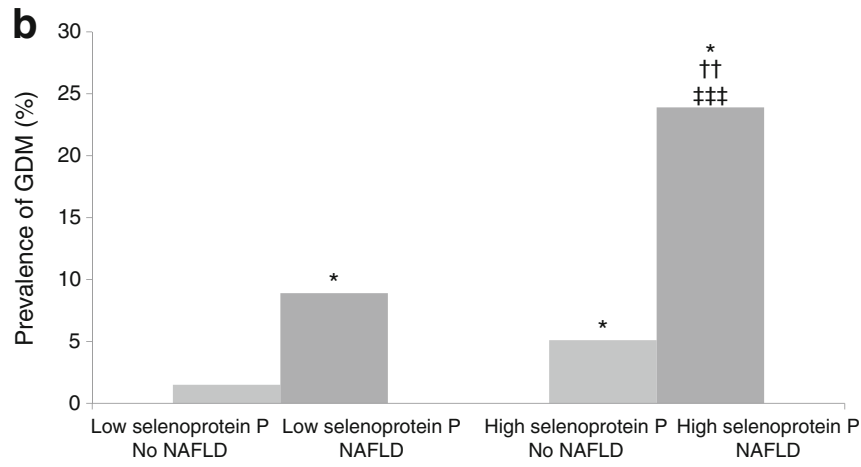

test. (b) The prevalence of GDM according to maternal plasma selenoprotein P and NAFLD status. High selenoprotein P is defined as $>50$ th percentile $(>6.08 \mu \mathrm{g} / \mathrm{ml})$ and low selenoprotein $\mathrm{P}$ is defined as $\leq 50$ th percentile. ${ }^{*} p<0.05$ vs low selenoprotein P without NAFLD; ${ }^{\dagger \dagger} p<0.005$ vs low selenoprotein P with NAFLD; ${ }^{\star+1} p<0.001 \mathrm{vs} \mathrm{high}$ selenoprotein P without NAFLD; all Mann-Whitney $U$ test 
We have also demonstrated that non-invasive steatosis indices (FLI and HSI) and maternal plasma adiponectin and selenoprotein $\mathrm{P}$ levels may be useful in predicting the risk of developing GDM. According to recently published clinical practice guidelines, early screening for diabetes is recommended in high-risk individuals who display metabolic risk factors such as severe obesity, a strong family history of type 2 diabetes, a previous history of GDM, impaired glucose tolerance or glycosuria [43]. In 2017, the American College of Obstetricians and Gynecologists adopted additional criteria for women at high risk, established by the American Diabetes Association: physical inactivity, high-risk race/ethnicity, previous history of macrosomia, hypertension, low levels of HDL-cholesterol, high levels of triacylglycerol and history of polycystic ovarian syndrome or cardiovascular disease [44]. Given that the OR (95\% CI) of NAFLD for GDM is $6.45(3.26,12.97)$ in the current study, our findings imply that NAFLD might be also considered a major risk factor for the development of GDM. Early identification of women with NAFLD and more intensive screening and preventive strategies such as lifestyle modifications are likely needed in this special population (i.e., pregnant women with NAFLD). However, the introduction of liver ultrasound into routine antenatal care during pregnancy might be reserved until cost-effective analysis of the clinical utility of liver ultrasound and large-scale, external validation of the role of NAFLD as a risk factor for GDM have been carried out.

The current study has some limitations. First, the diagnosis of NAFLD was based on liver ultrasound, FLI and HSI but not on histological examination. However, the histological confirmation of NAFLD does not seem to be feasible in asymptomatic pregnant women. Specifically, liver ultrasound is a weak diagnostic method for NAFLD, especially in the lower range of hepatic steatosis of $<10-15 \%$ [45]. Instead, in the current study, we demonstrated the relationship between NAFLD and GDM using both FLI and HSI, which are well-known indices for NAFLD. In addition, we also demonstrated that both FLI and HSI were positively correlated with the risk of GDM, even in women without fatty liver detected by ultrasound. Second, our study results should be validated in other ethnicities, because the relationship between NAFLD and GDM may vary depending on ethnicity.

In conclusion, the presence of NAFLD in early pregnancy is associated with an increased risk of developing GDM in the mid-gestation. Further mechanistic studies are needed to identify and characterise the underlying mechanisms responsible for this association.

Acknowledgements Some of the data were presented as an abstract at The International Liver Congress in 2018. The authors would like to thank S. Oh (the Department of Biostatistics, Seoul Metropolitan Government Seoul National University Boramae Medical Center, Korea) for statistical advice.
Data availability The data that support the findings of this study are available from the corresponding author in anonymised form upon reasonable request.

Funding This work was supported by a clinical research grant-in-aid from the Bio \& Medical Technology Development Program of the National Research Foundation (NRF) funded by the Ministry of Science and ICT of Korea (2016M3A9B6902061) and was also supported by the Korea Health Technology R\&D Project through the Korea Health Industry Development Institute (KHIDI) funded by the Ministry of Health \& Welfare, Republic of Korea (H I17C0912).

Duality of interest The authors declare that there is no duality of interest associated with this manuscript.

Contribution statement The study was conceived and designed by SML, WK and JSP. WK and JSP supervised the study. All authors acquired, analysed or interpreted data. SML carried out statistical analysis. SML, WK and JSP drafted the manuscript. All authors critically revised the manuscript for important intellectual content and approved the version to be published. SML, WK and JSP are guarantors who had full access to the data in the study and take responsibility for the integrity of the data and the accuracy of the data analysis.

\section{References}

1. Cho HC (2016) Prevalence and factors associated with nonalcoholic fatty liver disease in a nonobese Korean population. Gut Liver 10(1):117-125. https://doi.org/10.5009/gn114444

2. Choi SY, Kim D, Kim HJ et al (2009) The relation between nonalcoholic fatty liver disease and the risk of coronary heart disease in Koreans. Am J Gastroenterol 104(8):1953-1960. https://doi.org/10. 1038/ajg.2009.238

3. Vernon G, Baranova A, Younossi ZM (2011) Systematic review: the epidemiology and natural history of non-alcoholic fatty liver disease and non-alcoholic steatohepatitis in adults. Aliment Pharmacol Ther 34(3):274-285. https://doi.org/10.1111/j.13652036.2011.04724.x

4. Loomba R, Sanyal AJ (2013) The global NAFLD epidemic. Nat Rev Gastroenterol Hepatol 10(11):686-690. https://doi.org/10. 1038/nrgastro.2013.171

5. Ramesh S, Sanyal AJ (2005) Evaluation and management of nonalcoholic steatohepatitis. J Hepatol 42 Suppl(1):S2-12. https://doi. org/10.1016/j.jhep.2004.11.022

6. Targher G, Bertolini L, Padovani R et al (2007) Prevalence of nonalcoholic fatty liver disease and its association with cardiovascular disease among type 2 diabetic patients. Diabetes Care 30(5):12121218. https://doi.org/10.2337/dc06-2247

7. Marchesini G, Brizi M, Bianchi G et al (2001) Nonalcoholic fatty liver disease: a feature of the metabolic syndrome. Diabetes 50(8): 1844-1850. https://doi.org/10.2337/diabetes.50.8.1844

8. Das K, Das K, Mukherjee PS et al (2010) Nonobese population in a developing country has a high prevalence of nonalcoholic fatty liver and significant liver disease. Hepatology 51(5):1593-1602. https:// doi.org/10.1002/hep.23567

9. Amarapurkar D, Kamani P, Patel N et al (2007) Prevalence of nonalcoholic fatty liver disease: population based study. Ann Hepatol 6(3):161-163

10. Singh SP, Nayak S, Swain M et al (2004) Prevalence of nonalcoholic fatty liver disease in coastal eastern India: a preliminary ultrasonographic survey. Tropical gastroenterology : official journal of the Digestive Diseases Foundation 25(2):76-79

11. Koo BK, Joo SK, Kim D et al (2018) Additive effects of PNPLA3 and TM6SF2 on the histological severity of non-alcoholic fatty liver 
disease. J Gastroenterol Hepatol 33(6):1277-1285. https://doi.org/ 10.1111/jgh.14056

12. Tiikkainen M, Tamminen M, Hakkinen AM et al (2002) Liver-fat accumulation and insulin resistance in obese women with previous gestational diabetes. Obes Res 10(9):859-867. https://doi.org/10. 1038/oby.2002.118

13. Forbes S, Taylor-Robinson SD, Patel N, Allan P, Walker BR, Johnston DG (2011) Increased prevalence of non-alcoholic fatty liver disease in European women with a history of gestational diabetes. Diabetologia 54(3):641-647. https://doi.org/10.1007/ s00125-010-2009-0

14. Foghsgaard S, Andreasen C, Vedtofte L et al (2017) Nonalcoholic fatty liver disease is prevalent in women with prior gestational diabetes mellitus and independently associated with insulin resistance and waist circumference. Diabetes Care 40(1):109-116. https://doi. org/10.2337/dc16-1017

15. Ajmera VH, Gunderson EP, Vanwagner LB, Lewis CE, Carr JJ, Terrault NA (2016) Gestational diabetes mellitus is strongly associated with non-alcoholic fatty liver disease. Am J Gastroenterol 111(5):658-664. https://doi.org/10.1038/ajg.2016.57

16. Page LM, Girling JC (2011) A novel cause for abnormal liver function tests in pregnancy and the puerperium: non-alcoholic fatty liver disease. BJOG 118(12):1532-1535. https://doi.org/10.1111/j. 1471-0528.2011.03070.x

17. Sharma DL, Lakhani HV, Klug RL et al (2017) Investigating molecular connections of non-alcoholic fatty liver disease with associated pathological conditions in West Virginia for biomarker analysis. J Clin Cell Immunol 8(5). https://doi.org/10.4172/2155-9899. 1000523

18. Misu H, Takamura T, Takayama $\mathrm{H}$ et al (2010) A liver-derived secretory protein, selenoprotein $\mathrm{P}$, causes insulin resistance. Cell Metab 12(5):483-495. https://doi.org/10.1016/j.cmet.2010.09.015

19. Ewing JA (1984) Detecting alcoholism. The CAGE questionnaire. JAMA 252(14):1905-1907. https://doi.org/10.1001/jama.1984. 03350140051025

20. Saadeh S, Younossi ZM, Remer EM et al (2002) The utility of radiological imaging in nonalcoholic fatty liver disease. Gastroenterology 123(3):745-750. https://doi.org/10.1053/gast. 2002.35354

21. Taylor KJ, Riely CA, Hammers L et al (1986) Quantitative US attenuation in normal liver and in patients with diffuse liver disease: importance of fat. Radiology 160(1):65-71. https://doi.org/10. 1148/radiology.160.1.3520657

22. Bedogni G, Bellentani S, Miglioli L et al (2006) The fatty liver index: a simple and accurate predictor of hepatic steatosis in the general population. BMC Gastroenterol 6(1):33. https://doi.org/10. 1186/1471-230X-6-33

23. Gastaldelli A, Kozakova M, Hojlund K et al (2009) Fatty liver is associated with insulin resistance, risk of coronary heart disease, and early atherosclerosis in a large European population. Hepatology 49(5):1537-1544. https://doi.org/10.1002/hep.22845

24. Bozkurt L, Gobl CS, Tura A et al (2012) Fatty liver index predicts further metabolic deteriorations in women with previous gestational diabetes. PLoS One 7(2):e32710. https://doi.org/10.1371/journal. pone.0032710

25. Lee JH, Kim D, Kim HJ et al (2010) Hepatic steatosis index: a simple screening tool reflecting nonalcoholic fatty liver disease. Dig Liver Dis 42(7):503-508. https://doi.org/10.1016/j.dld.2009. 08.002

26. Matthews DR, Hosker JP, Rudenski AS, Naylor BA, Treacher DF, Turner RC (1985) Homeostasis model assessment: insulin resistance and beta-cell function from fasting plasma glucose and insulin concentrations in man. Diabetologia 28(7):412-419. https://doi.org/ 10.1007/BF00280883
27. Committee on Practice B-O (2013) Practice Bulletin No. 137: gestational diabetes mellitus. Obstet Gynecol 122:406-416

28. Carpenter MW, Coustan DR (1982) Criteria for screening tests for gestational diabetes. Am J Obstet Gynecol 144(7):768-773. https:// doi.org/10.1016/0002-9378(82)90349-0

29. Firth D (1993) Bias reduction of maximum likelihood estimates. Biometrika 80(1):27-38. https://doi.org/10.1093/biomet/80.1.27

30. Ryu S, Suh BS, Chang Y et al (2015) Menopausal stages and nonalcoholic fatty liver disease in middle-aged women. Eur J Obstet Gynecol Reprod Biol 190:65-70. https://doi.org/10.1016/j.ejogrb. 2015.04.017

31. Lee JY, Shin DW, Oh JW et al (2017) Non-alcoholic fatty liver disease as a risk factor for female sexual dysfunction in premenopausal women. PLoS One 12(8):e0182708. https://doi.org/10.1371/ journal.pone. 0182708

32. De Souza LR, Berger H, Retnakaran R et al (2016) Non-alcoholic fatty liver disease in early pregnancy predicts dysglycemia in midpregnancy: prospective study. Am J Gastroenterol 111(5):665-670. https://doi.org/10.1038/ajg.2016.43

33. Hagstrom H, Hoijer J, Ludvigsson JF et al (2016) Adverse outcomes of pregnancy in women with non-alcoholic fatty liver disease. Liver Int 36(2):268-274. https://doi.org/10.1111/liv.12902

34. Mousa N, Abdel-Razik A, Shams M et al (2018) Impact of nonalcoholic fatty liver disease on pregnancy. Br J Biomed Sci 75:197199

35. Mccullough AJ (2004) The clinical features, diagnosis and natural history of nonalcoholic fatty liver disease. Clin Liver Dis 8:521533 viii

36. Porepa L, Ray JG, Sanchez-Romeu P, Booth GL (2010) Newly diagnosed diabetes mellitus as a risk factor for serious liver disease. CMAJ 182(11):E526-E531. https://doi.org/10.1503/cmaj.092144

37. Despres JP (1993) Abdominal obesity as important component of insulin-resistance syndrome. Nutrition 9(5):452-459

38. Meng K, Lee CH, Saremi F (2010) Metabolic syndrome and ectopic fat deposition: what can CT and MR provide? Acad Radiol 17(10):1302-1312. https://doi.org/10.1016/j.acra.2010.03.019

39. Larter CZ, Chitturi S, Heydet D, Farrell GC (2010) A fresh look at NASH pathogenesis. Part 1: the metabolic movers. J Gastroenterol Hepatol 25(4):672-690. https://doi.org/10.1111/j.1440-1746.2010. 06253.x

40. Lebensztejn DM, Flisiak-Jackiewicz M, Bialokoz-Kalinowska I, Bobrus-Chociej A, Kowalska I (2016) Hepatokines and nonalcoholic fatty liver disease. Acta Biochim Pol 63(3):459-467. https://doi.org/10.18388/abp.2016_1252

41. Kim JY, Park KJ, Hwang JY et al (2017) Activating transcription factor 3 is a target molecule linking hepatic steatosis to impaired glucose homeostasis. J Hepatol 67(2):349-359. https://doi.org/10. 1016/j.jhep.2017.03.023

42. Misu H, Ishikura K, Kurita S et al (2012) Inverse correlation between serum levels of selenoprotein $\mathrm{P}$ and adiponectin in patients with type 2 diabetes. PLoS One 7(4):e34952. https://doi.org/10. 1371/journal.pone.0034952

43. Metzger BE, Buchanan TA, Coustan DR et al (2007) Summary and recommendations of the Fifth International Workshop-Conference on Gestational Diabetes Mellitus. Diabetes Care 30 (Suppl 2): S251-S260. https://doi.org/10.2337/dc07-s225

44. Committee on Practice B-O (2018) ACOG Practice Bulletin No. 190: gestational diabetes mellitus. Obstet Gynecol 131:e49-e64

45. Bril F, Ortiz-Lopez C, Lomonaco R et al (2015) Clinical value of liver ultrasound for the diagnosis of nonalcoholic fatty liver disease in overweight and obese patients. Liver Int 35(9):2139-2146. https://doi.org/10.1111/liv.12840 